\title{
The impact of a heat and moisture exchange mask on respiratory symptoms and airway response to exercise in asthma
}

\author{
Anna R. Jackson ${ }^{1,2}$, James H. Hull (i) $2,3,4$, James G. Hopker ${ }^{1}$, Hannah Fletcher ${ }^{5}$, \\ William Gowers ${ }^{1}$, Surinder S. Birring ${ }^{5}$ and John W. Dickinson ${ }^{1}$
}

Affiliations: ${ }^{1}$ School of Sport and Exercise Sciences, University of Kent, Chatham, UK. ${ }^{2}$ English Institute of Sport, London, UK. ${ }^{3}$ Dept of Respiratory Medicine, Royal Brompton Hospital, London, UK. ${ }^{4}$ National Heart and Lung Institute, Imperial College, London, UK. ${ }^{5}$ King's College Hospital NHS Foundation Trust, London, UK.

Correspondence: John W. Dickinson, School of Sport and Exercise Sciences, University of Kent, Medway Building, Chatham, Kent, ME4 4AG, UK. E-mail: j.w.dickinsonakent.ac.uk

ABSTRACT Respiratory symptoms, including cough, are prevalent in individuals with asthma when exercising. This study investigates whether a heat and moisture exchanger (HME) face mask is effective in modulating exercise-induced bronchoconstriction (EIB) and post-exercise cough in a cold, dry environment in individuals with asthma.

Twenty-six participants diagnosed with asthma (20 males, 6 females) completed three cycling exercise challenges at $8^{\circ} \mathrm{C}$ and $24 \%$ relative humidity in a randomised order. Participants wore either an HME mask (MASK), sham mask (SHAM), or no mask (CONT). Following a 3-min warm-up, participants completed 6-min cycling at $80 \%$ peak power output. Before and after exercise, maximal flow-volume loops were recorded. Post-exercise cough was monitored with a Leicester Cough Monitor (LCM) for $24 \mathrm{~h}$. Results were analysed using repeated-measures ANOVA and Friedman's tests and data were presented as the mean \pm SD or median (interquartile range (IQR)).

Eleven participants failed to demonstrate EIB (i.e. $>10 \%$ fall in forced expiratory volume in $1 \mathrm{~s}$ after exercise) and were removed from analysis. The percentage fall in forced expiratory volume in $1 \mathrm{~s}$ following exercise in CONT was greater than MASK (MASK: $-6 \%$ (7\%), SHAM: $-11 \%(11 \%)$, CONT: $-13 \%(9 \%)$; $\mathrm{p}<0.01)$. No difference was found between exercise in cough count per hour over the 24-h monitoring period or the number of coughs in the first hour after exercise.

HME masks can attenuate EIB when exercising in cold, dry environments. The SHAM mask may not have been entirely inert, demonstrating the challenges of running randomised control trials utilising control and sham conditions.

@ERSpublications

Heat and moisture exchanger masks can reduce bronchoconstriction in individuals with exercise-induced bronchoconstriction when exercising in cold, dry environments https://bit.ly/ 2JKeLnX

Cite this article as: Jackson AR, Hull JH, Hopker JG, et al. The impact of a heat and moisture exchange mask on respiratory symptoms and airway response to exercise in asthma. ERJ Open Res 2020; 6: 00271-2019 [https://doi.org/10.1183/23120541.00271-2019].

Data available from the Dryad Digital Repository: https:/doi.org/10.5061/dryad.gf1vhhmkm.

ClinicalTrials.gov identifier: NCT04302610.

Received: 1 Oct 2019 | Accepted after revision: 31 March 2020 | First published: 22 June 2020

Copyright $\odot$ ERS 2020. This article is open access and distributed under the terms of the Creative Commons Attribution Non-Commercial Licence 4.0. 


\section{Introduction}

Inspiring dry and cold air during moderate and vigorous physical activity is the key trigger for bronchoconstriction, precipitating dehydration of the airway surface liquid and leading to cell shrinkage and release of inflammatory mediators and airway smooth muscle constriction [1]. This respiratory water loss and resultant airway surface mucosal drying is also thought to lead to both physical and chemical activation of cough receptors [2].

Repeated exercise in the cold is thought to result in a continuous cycle of airway injury and repair, leading to chronically inflamed airways, epithelial damage and cellular airway change [3]. These modifications to airway structure and function appear to underpin the heightened prevalence of exercise-induced bronchoconstriction (EIB) and cough observed in endurance athletes who train and compete in cold and dry environments [4].

It has been known for some time that an increase in the temperature and water content of inspired air can attenuate EIB in asthmatic subjects [5, 6]. More recently, BolgER et al. [7] demonstrated that EIB in athletes was completely prevented by increasing the temperature and water content of an inspirate from $4^{\circ}$ C, $37 \%$ relative humidity to $25^{\circ} \mathrm{C}, 94 \%$ relative humidity. Post-exercise cough also seems to be more prevalent in environmental conditions that promote airway heat and water loss [2].

One method of increasing the temperature of inspired air and thereby potentially diminishing airway dehydration is to use a facemask that incorporates a heat and moisture exchanger (HME); [8-11]. An HME mask will warm and humidify inspired air and therefore reduce airway heat and water loss during exercise. This will potentially reduce the incidence of and severity of EIB and may also have the potential to decrease the incidence of cough among athletes engaging in sports in cold dry environments [12-14]. This is important, given the emphasis placed on nonpharmacological treatment options by patients with asthma. Despite this, to date, international guideline documents [15] indicate that the evidence in support of using HME masks is weak based, on the current availability of only low-quality randomised and noncontrol-based evidence.

The aim of this study was to address this deficiency in the literature by undertaking a randomised SHAM controlled study in asthmatic individuals with EIB, to determine whether an HME facemask can protect against acute bronchoconstriction and, for the first time, post-exercise cough in response to a cycling exercise challenge in a cold, dry environment.

\section{Methods}

\section{Study population}

This study is registered on ClinicalTrials.gov (identifier: NCT04302610). Following approval from the Faculty of Sciences Research Ethics Advisory Group for Human Participants, University of Kent (0881516) 34 recreationally active participants exercising at least twice per week $(6 \pm 2 \mathrm{~h})$ provided written informed consent to participate. All participants had a clinician-based diagnosis of asthma; however, participants who did not have a fall in forced expiratory volume in $1 \mathrm{~s}\left(\mathrm{FEV}_{1}\right)$ of $\geqslant 10 \%$ at two consecutive time points following at least one of the exercise challenges (see below) were not included in subsequent analysis (i.e. no evidence of EIB).

Participants were excluded if they used an oral corticosteroid daily, were hospitalised due to asthma in the 6 months prior to study commencement and/or had resting $\mathrm{FEV}_{1}<80 \%$ of predicted value [16]. All participants were free from illness in the 2 weeks prior to assessment. Participants were instructed to maintain their usual diet for the duration of the study, to avoid exercise and caffeine for $24 \mathrm{~h}$ and $4 \mathrm{~h}$ respectively before each visit and arrive at the laboratory at least $2 \mathrm{~h}$ post-prandial.

\section{Study design}

In a randomised crossover design, participants attended the laboratory on five occasions (figure 1). The visits were as follows: visit 1 , peak oxygen uptake (peak $V^{\prime} \mathrm{O}_{2}$ ) test on a cycle ergometer; visit 2, familiarisation, and visits 3-5, standardised cycle exercise challenge (EX) in a cold, dry environment. During exercise, participants wore either an HME mask (MASK) (ColdAvenger ${ }^{\circledast}$ expedition balaclava, www.coldavenger.com), a sham mask (SHAM) that was the same HME mask with holes cut across the entire ventilator cup and the ventilator removed (figure 2), or no mask (CONT), wearing only the balaclava to which the mask is attached.

The exercise challenges were completed in a randomised order but at the same time of day. The time between each visit was dependant on the participant's current medication; participants previously prescribed inhaler medication for asthma/EIB withheld medication prior to each assessment (inhaled corticosteroids (ICSs): $72 \mathrm{~h}$; inhaled long-acting $\beta_{2}$-agonists (LABAs): $48 \mathrm{~h}$; inhaled short-acting $\beta_{2}$-agonists (SABAs): the day of the test) [17]. Following each trial, participants had the same amount of 


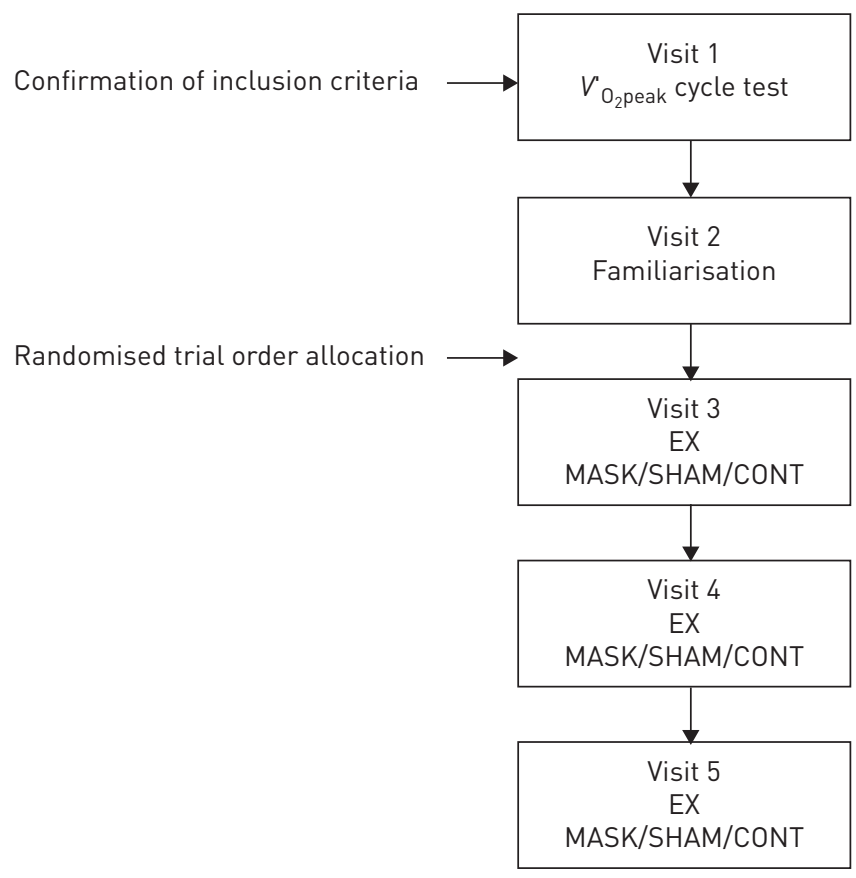

FIGURE 1 Flow chart of study design. $V^{\prime}{ }_{O_{2} \text { peak: }}$ peak oxygen uptake; EX: exercise; MASK: heat and moisture exchanger mask; SHAM: sham mask; CONT: no mask.

time back on their medication before once again stopping treatment before their next exercise. Participants who were not taking any asthma medication, had a minimum of $48 \mathrm{~h}$ between trials.

\section{Visit details}

\section{Visit one}

Cough-specific health status was assessed with the Leicester Cough Questionnaire (LCQ), which is a self-administered 19-item tool (total score range 3-21; with higher scores indicating better health status; [18]). Anthropometric measures were taken and a standardised incremental ramp test to volitional exhaustion was performed to establish peak power on a cycle ergometer (Lode; Corival, Groningen,

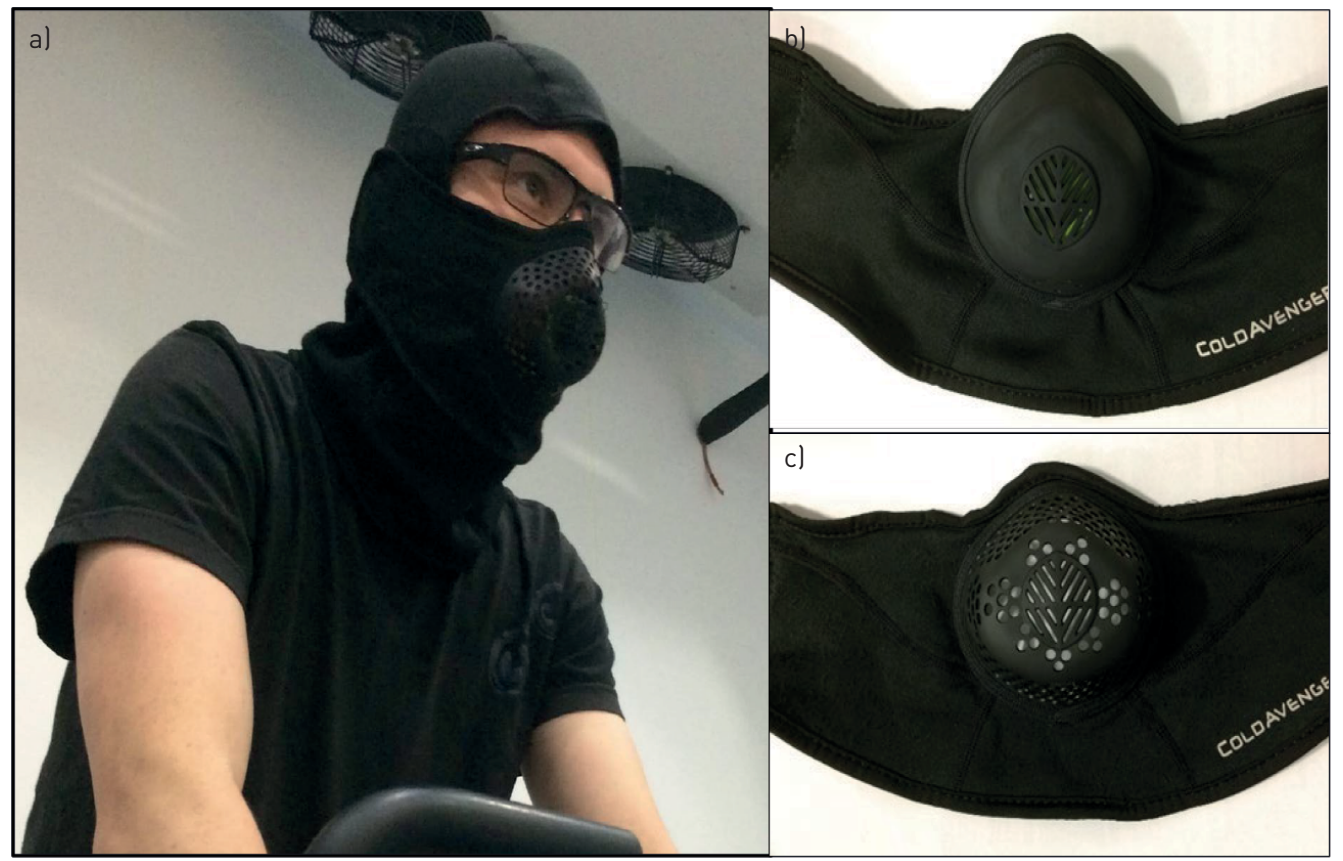

FIGURE 2 a) Participant wearing the sham mask during exercise. b) Heat and moisture exchanger mask. c) Sham mask. 
Netherlands) with simultaneous gas analysis (Cortex Metalyser 3b, CORTEX Biophysik GmbH, Germany). Heart rate was recorded throughout (Polar RS400; Polar Electro Oy, Kempele, Finland) and peak power output was recorded.

Visit two

Participants remained on prescribed asthma therapy and completed the exercise protocol as detailed below in a normal lab environment without a mask, as a means of laboratory testing familiarisation.

Visits three to five

Participants completed a cough $0-100 \mathrm{~mm}$ visual analogue scale (VAS) [19]. Airway inflammation was then assessed prior to each challenge by determining the fraction of exhaled nitric oxide $\left(F_{\mathrm{eNO}}\right)$ (NIOX VERO, NIOX, Aerocrine, Sweden) [20]. Resting lung function was then measured by maximal flow-volume spirometry (Spiro-USB and MicroLab, CareFusion, Germany) in accordance with international standards [21]. Maximal flow-volume loops were subsequently measured in duplicate at 3, 5, 7, 10 and $15 \mathrm{~min}$ after the challenge, with the highest value at each time point used for analysis. If there was a $\geqslant 10 \%$ fall in $\mathrm{FEV}_{1}$ post-challenge at two consecutive time points, $400 \mu \mathrm{g}$ inhaled salbutamol was self-administered by the participant and maximal flow loops were repeated 15 min post-administration to ensure that $\mathrm{FEV}_{1}$ had returned to within $10 \%$ of baseline. The exercise challenges were conducted in an environmental chamber (TIS Services, Hampshire, UK) $\left(8.6 \pm 0.9^{\circ} \mathrm{C}, 24.2 \pm 4.2 \%\right.$ relative humidity) on a cycle ergometer (Lode; Corival, Groningen, Netherlands).

The exercise protocol required participants to complete $3 \mathrm{~min}$ of incremental cycling at a work rate of 60 , 75 and $90 \%$ of their final target power for $1 \mathrm{~min}$ at each power output. They then cycled for $6 \mathrm{~min}$ at $80 \%$ of their peak power (CRAPO et al. [22, 23]). Heart rate was recorded throughout.

Immediately after exercise, cough frequency was assessed objectively over $24 \mathrm{~h}$ with the validated LCM [24, 25]. The LCM is an ambulatory system that comprises an MP3 recorder (ICD-PX333, Sony Corporation, Tokyo, Japan), a lapel free-field microphone (LFH9173, Philips, Amsterdam, the Netherlands) and semi-automated cough detection software. Coughs were detected as single events whether they occurred in isolation or in bouts [25]. Cough data were analysed via cough detection software based on the hidden Markov model as described previously [25]. Participants completed an additional VAS $24 \mathrm{~h}$ after exercise.

\section{Statistical analysis}

Data are presented as mean \pm SD unless otherwise stated. Shapiro-Wilk tests were used to test for normal distribution. Differences between the three exercise conditions were examined using repeated-measures ANOVA. Where data were not normally distributed, Friedman's test was used with post hoc pairwise comparisons where appropriate. Spearman's rank correlation coefficient was used to investigate the relationship between VAS score and cough per hour, percentage fall in $\mathrm{FEV}_{1}$ and coughs per hour after exercise or LCQ score and cough count. All analysis was conducted using SPSS software, v.23 (SPSS, IBM, Armonk, NY, USA) with significance accepted at $\mathrm{p}<0.05$.

\section{Results}

Thirty-four recreationally active participants were initially enrolled in the study. Two participants were lost to subsequent follow-up following the peak $V_{\mathrm{O}_{2}}^{\prime}$ trial (three following the familiarisation and two following the initial exercise). One participant was excluded due to impaired resting lung function $\left(\mathrm{FEV}_{1}<70 \%\right.$ predicted at baseline). The remaining 26 participants $(20$ males, 6 females, aged $27.6 \pm 9.2$ years, height: $172.7 \pm 7.3 \mathrm{~cm}$, mass: $71.2 \pm 12.8 \mathrm{~kg}$, exercising: $5.8 \pm 2.2 \mathrm{~h}$ per week, peak $V^{\prime} \mathrm{O}_{2}$ : $42.8 \pm 8.2 \mathrm{~mL} \cdot \mathrm{kg}^{-1} \cdot \mathrm{min}^{-1}$ ). Seventeen patients were prescribed inhaler therapy for their asthma (seven ICSs, four, combined ICSs and LABAs and eight SABAs as required). Eleven of the 26 participants however, failed to demonstrate a fall $<10 \%$ in $\mathrm{FEV}_{1}$ after exercise in any of the trials (range, 4 to $-9 \%$ ) and thus we were unable to confirm definite evidence of asthma/EIB. Of these, three had a history of EIB only, three were currently using ICSs, two were using a combination inhaler, two were using only SABAs and four were using no therapy. Subsequent analysis was thus only undertaken using the participants (12 males, 3 females) with confirmatory evidence of EIB (table 1).

\section{Exercise trials}

Descriptive data for lung function pre and post-challenge can be seen in table 2. The percentage fall in $\mathrm{FEV}_{1}$ following the CONT exercise was significantly greater than that of MASK $(\mathrm{p}<0.01)$, with all but one participant demonstrating a greater fall in $\mathrm{FEV}_{1}$ following CONT than MASK (figure 3). Ten out of 15 participants also had a greater drop in SHAM than MASK (figure 3); however, SHAM was not significantly different from either MASK $(p=0.17)$ or CONT $(p=0.51)$. 


\section{TABLE 1 Participant characteristics, peak oxygen uptake $\left(V^{\prime}{ }_{O_{2}}\right)$ and baseline respiratory} assessment data (while using current medication) ( $n=15)$

\section{Measured}

$29.3 \pm 9.2$

Age years

Height $\mathrm{cm}$

Weight kg

Peak $V^{\prime} \mathrm{O}_{2}$

Peak power output $\mathrm{W}$

FEV 1 L

FVC L

PEF L. $\min ^{-1}$

FEV $_{1} /$ FVC
$172.2 \pm 8.1$

$73.4 \pm 14.0$

$43.26 \pm 8.11$

$246.3 \pm 42.7$

$3.57 \pm 0.64$

$4.67 \pm 0.87$

$536 \pm 93$

$76.7 \pm 8.8$
Percentage of predicted

Data are presented as mean $\pm S D$. FEV ${ }_{1}$ : forced expiratory volume in $1 \mathrm{~s}$; FVC: forced vital capacity; PEF: peak expiratory flow.

\section{TABLE 2 Lung function pre and post-challenge (n=15)}

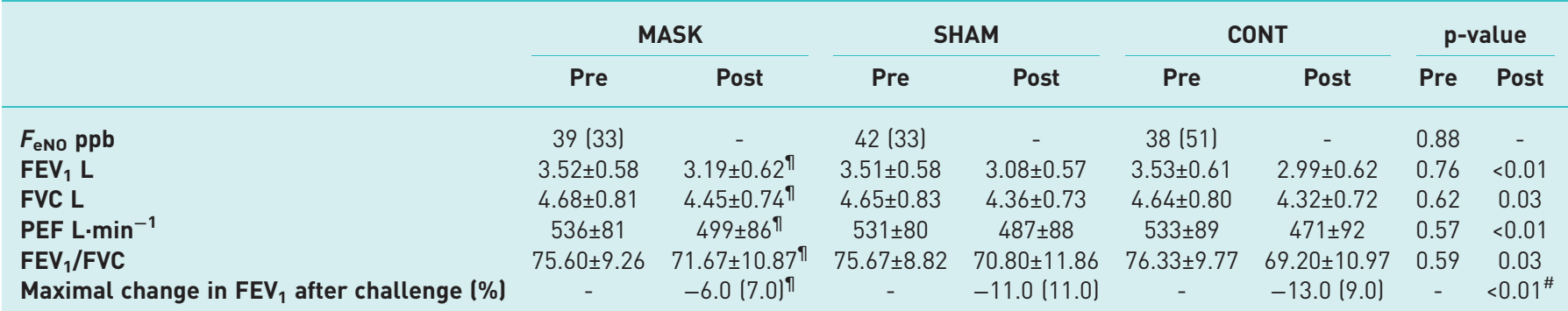

Data for maximal percentage change in $\mathrm{FEV}_{1}$ and $F_{\mathrm{eNO}}$ were not normally distributed and therefore were analysed with Friedman's tests and presented as the median score (interquartile range). Other data are presented as mean $\pm S D$, unless otherwise stated. MASK: heat and moisture exchanger mask; SHAM: sham mask; CONT: no mask; $F_{\text {eNo }}$ : exhaled nitric oxide fraction; $\mathrm{FEV}_{1}$ : forced expiratory volume in $1 \mathrm{~s} ; \mathrm{FVC}$ : forced vital capacity; PEF: peak expiratory flow. ${ }^{\#}$ : significant difference $(p<0.05)$ between exercise condition; ${ }^{\Uparrow}$ : significant difference ( $\left.p<0.05\right)$ versus CONT.

FIGURE 3 Maximum percentage fall from baseline in forced expiratory volume in $1 \mathrm{~s}\left(\mathrm{FEV}_{1}\right)$ after exercise challenge. MASK: heat and moisture exchanger mask: SHAM: sham mask; CONT: no mask. $*$ : significant difference $(p<0.05)$ versus CONT. Dashed line represents the threshold for a positive challenge.
One participant did not participate in cough monitoring due to their occupation. Four of the remaining 14 participants (29\%) reported cough as a problematic symptom following exercise.

The LCQ showed all but one participant scored either a 6 (hardly any of the time) or 7 (none of the time) in all domains (physical, psychological and social) regarding the impact that cough had upon the different

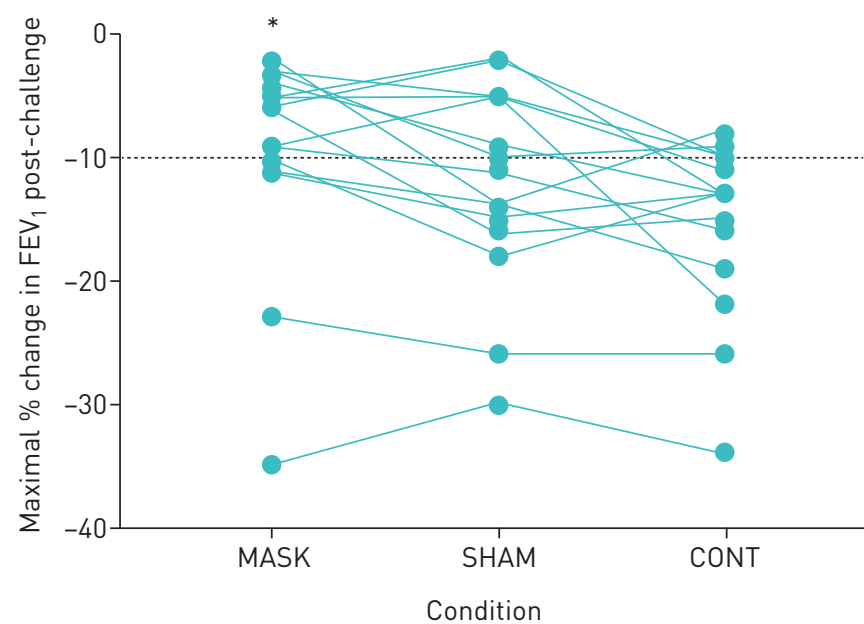


FIGURE 4 Coughs per hour over a 24-h monitoring period. MASK: heat and moisture exchanger mask; SHAM: sham mask; CONT: no mask.

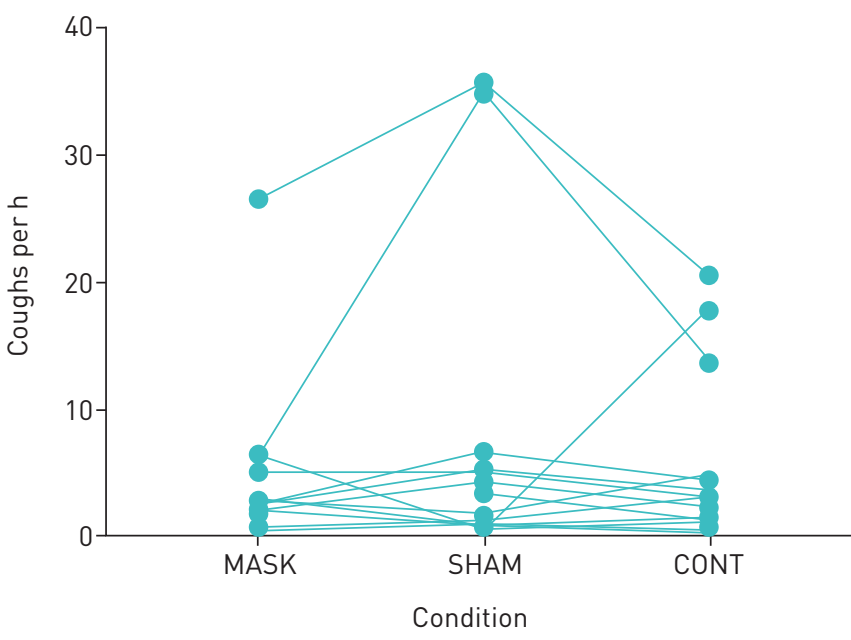

aspects of their lives. The remaining participant scored 5 (a little of the time) for all domains. No difference was seen in LCQ total score between those who reported cough as a troublesome symptom (median, 19; IQR, 3) and those who did not (median, 20; IQR, 2).

Baseline cough VAS score was $9 \pm 7 \mathrm{~mm}$ out of $100 \mathrm{~mm}$, with a range of 1 to $19 \mathrm{~mm}$. No differences were seen between exercise trials in cough count per hour over $24 \mathrm{~h}$ (figure 4), number of coughs recorded in the first hour following exercise or participants self-report of cough $24 \mathrm{~h}$ after exercise trial using VAS (table 3).

There was no difference between those who reported cough as a troublesome symptom and those who did not in the number of coughs in the first hour ( $\mathrm{n}=14$, yes cough: $18 \pm 14$, no cough: $13 \pm 13, \mathrm{p}=0.56$ ) or cough per hour in the 24 - $h$ monitoring period ( $n=14$, yes cough: $2 \pm 2$, no cough: $3 \pm 2, p=0.88$ ), in the familiarisation visit or any of the experimental conditions.

Spearman's rank correlation coefficient demonstrated no relationship between VAS score and cough per hour, percentage fall in $\mathrm{FEV}_{1}$ and coughs per hour after exercise or LCQ score and cough count following the familiarisation trial.

\section{Discussion}

This study demonstrates that wearing an HME mask during a cycle exercise challenge in a cold, dry environment results in an attenuation in EIB severity when compared with not wearing a mask. Only, a third of participants with EIB demonstrated evidence of EIB ( $\geqslant 10 \%$ fall in $\mathrm{FEV}_{1}$ after exercise) while wearing a mask, compared to $87 \%$ with no mask.

The results from the present study are in agreement with earlier studies: STEWART et al. [12] concluded that their facemask retained heat and moisture and effectively controlled EIB in most patients following exercise at $80 \%$ predicted $\mathrm{HR}_{\max }$. They noted that wearing the mask decreased the post-exercise drop in $\mathrm{FEV}_{1}$ by $9 \%$. The mask in our study was more effective in controlling EIB; however, without the mask, their patients exhibited a larger range in EIB severity ( $\mathrm{FEV}_{1}$ fall post-exercise between 18 to $52 \%$ ). Similarly, NISAR et al. [11] demonstrated that wearing an active mask during a 6-min cycle challenge inhaling a cold air supply $\left(-13^{\circ} \mathrm{C}\right)$ reduced the post-exercise fall in $\mathrm{FEV}_{1}$ to $-10 \%$ compared to $-22 \%$ with a control mask.

\begin{tabular}{lcccc} 
TABLE 3 Cough results $(n=13)$ & & & & \\
& MASK & SHAM & CONT & p-value \\
\hline Number of coughs per hour & $3(3)$ & $2(3)$ & $3(3)$ & 0.06 \\
Number of coughs in first hour after exercise & $17(17)$ & $15(17)$ & $8(24)$ & 0.92 \\
VAS 24-h after exercise mm $(n=15)$ & $7(24)$ & $14(27)$ & $10(24)$ & 0.52
\end{tabular}

Data were not normally distributed and therefore were analysed with Friedman's test and presented as the median score (interquartile range). MASK: heat and moisture exchanger mask; SHAM: sham mask; CONT: no mask. VAS: 0-100 mm visual analogue scale. 
Previous studies have also compared HME masks to pre-treatment with a bronchodilator and found that they were equally effective in attenuating a drop in lung function $[8,10]$. BEUTHER AND MARTIN [8] reported that following 10 min running at $85 \%$ HRmax, breathing cold medical grade air ( -15 to $\left.-25^{\circ} \mathrm{C}\right), \mathrm{FEV}_{1}$ fell $28 \%$ with a placebo mask, $6 \%$ with an active mask, and $11 \%$ with pre-treatment with albuterol. Furthermore, combining the use of inhaled $\beta_{2}$-agonists prior to exercise with an HME mask may completely preserve lung function [10]. A benefit to this strategy may be that it can provide complete control against various mechanisms underlying EIB. Future investigations could investigate the long-term impact of using inhaler therapy and HME in combination to protect against EIB and maintain airway health.

Acute exercise in cold, dry environments increases the risk of developing EIB [6]. It has been suggested that the use of $\beta_{2}$-agonists may make it possible for more cold, dry air to reach the lower airways. Although the $\beta_{2}$-agonists will acutely reduce bronchoconstriction, they may in the end cause more injury to the mucosa [10]. This combined with the effectiveness of the HME mask suggests that there may be value in encouraging all individuals exercising in cold, dry environments who are susceptible to EIB, to wear an HME mask where practical, whether they are using medication or not. Another potential benefit of using an HME for the prevention of EIB is that it has the potential to reduce the level of $\beta_{2}$-agonists that athletes use prophylactically, which would be beneficial as there is the potential for the development of tachyphylaxis and a potential desensitisation of repeat dosing [26].

As a group, our participants demonstrated a benefit to wearing the HME mask when compared with a control condition. However, two of our participants did not appear to respond to wearing the HME mask. These participants had the most severe EIB of the group. It is not clear whether this was related to EIB severity or phenotype as it was not feasible to conduct a subanalysis between mild and moderate EIB participants due to relatively small number of participants in each severity classification. We encourage future research to investigate the differences in response to HME masks between EIB severity and phenotype.

This was the first study to attempt to measure objective and subjective measures of cough following exercise in participants with EIB. Recently, the LCM has proven useful in objectively assessing cough in asthma management [27] and an alternative automated device has also been found capable of increasing patient awareness of the patterns of cough for early detection of worsening asthma [28]. These studies show that cough monitoring has great potential for assessing the response to asthma therapy. Results from this study however, showed no differences in cough frequency following exercise between trials for either cough per hour over the 24-h monitoring period, or more acutely in the hour after exercise. In addition, no differences were seen in the participant's perception of cough, as indicated by VAS. This however, is unsurprising because the relationship between objective cough frequency and subjective measures of cough such as VAS has been shown to be mild to moderate [24]. Furthermore, as per guidelines [15] we adhered to restricting ICSs for $72 \mathrm{~h}$ but it is possible that a longer (i.e. $>2$ weeks) ICS washout may result in a higher background coughing frequency.

Surprisingly, only four participants in this study reported cough as a troublesome symptom following exercise. It may be that cough frequency in the current cohort was too small to observe any change. Low levels of cough were unexpected, given that cough is the most commonly reported respiratory symptom by athletes [29]. Participants in this study were recreational rather than elite athletes so this may go some way to explain this observation. The environment we subjected our participants to may also have not been provocative enough to induce cough. A recent study demonstrated that $68 \%$ of participants who exercised at $-20^{\circ} \mathrm{C}$ experienced significant cough, which was significantly attenuated when participants exercised wearing an HME mask [14].

A prior power calculation indicated that we needed to recruit 34 participants with EIB. Despite recruiting 34 participants, the final number reduced by $55 \%$ due to participant withdrawal and others failing to demonstrate evidence of EIB during the trials. All participants had a physician's diagnosis of asthma, and the majority were taking prescribed medication; however, 11 out of $26(42 \%)$ demonstrated no evidence of EIB during the trial. This is an important issue by itself and is in support of the study in which AARON et al. [30] found that in $33.1 \%$ of adults with physician-diagnosed asthma, evidence of asthma could not be established. This highlights that objective testing should be utilised more frequently in the diagnosis and follow-up of asthma patients.

Furthermore, this impacted on the power of the study and under-recruiting participants with EIB may be the reason that there was no statistical difference between the HME mask and SHAM conditions. However, the difference between the HME mask and SHAM fall in $\mathrm{FEV}_{1}$ was $5 \%$, which may be clinically meaningful, especially as the mask reduced the mean fall below the $10 \%$ fall criterion to demonstrate EIB. Many of the earlier studies only included the use of either a sham or a no mask trial, and we felt it was 
important to include both. There may also be the possibility that the SHAM still provided some warming and humidification to the inspired air, and therefore offered some protection. Indeed, an earlier study demonstrated partial attenuation of EIB when individuals exercised in cold environments and covered their mouth with a scarf [31]. Therefore, it may be beneficial for athletes who may not want to wear an actual mask to cover their nose and mouth with a scarf or snood during training and competing in cold environments.

As seen in figure 2, to wear the mask we used it needs to be attached to a balaclava. Participants reported that they became very hot even in a short exercise bout, which would potentially be a barrier to athletes wearing the mask during training. Future investigations may look to develop a heat and moisture mask that is more practical to wear in UK weather or investigate whether similar benefits can be achieved through using an everyday scarf to cover the mouth during exercise.

In conclusion, HME masks can attenuate bronchoconstriction in individuals with asthma/EIB when exercising in cold, dry environments. Developments in mask design should look to make the masks less intrusive on the individual so that they are more likely to wear them during exercise in dry and cold environments.

Acknowledgements: The authors thank John Molphy (Research Institute for Sport and Exercise Sciences, Liverpool John Moores University, Liverpool, UK) with his assistance in data collection.

Conflict of interest: A.R. Jackson has nothing to disclose. J.H. Hull has nothing to disclose. J.G. Hopker has nothing to disclose. H. Fletcher has nothing to disclose. W. Gowers has nothing to disclose. S.S. Birring reports grants and personal fees from Merck, and personal fees from Bayer, NeRRe and Sanofi, outside the submitted work. J.W. Dickinson has nothing to disclose.

Support statement: This study was funded by AsthmaUK. Funding information for this article has been deposited with the Crossref Funder Registry.

\section{References}

1 Kippelen P, Anderson SD. Pathogenesis of exercise-induced bronchoconstriction. Immunol Allergy Clin North Am 2013; 33: 299-312.

2 Banner AS, Green J, O'Connor M. Relation of respiratory water loss to coughing after exercise. N Engl J Med 1984; 311: 883-886.

3 Karjalainen EM, Laitinen A, Sue-Chu M, et al. Evidence of airway inflammation and remodeling in ski athletes with and without bronchial hyperresponsiveness to methacholine. Am J Respir Crit Care Med 2000; 161: 2086-2091.

4 Turmel J, Bougault V, Boulet L-P. Seasonal variations of cough reflex sensitivity in elite athletes training in cold air environment. Cough 2012; 8: 1-8.

5 Anderson SD, Daviskas E, Schoeffel RE, et al. Prevention of severe exercise-induced asthma with hot humid air. Lancet 1979; 314: 629 .

6 Anderson SD, Kippelen P. Airway injury as a mechanism for exercise-induced bronchoconstriction in elite athletes. J Allergy Clin Immunol 2008; 122: 225-235.

7 Bolger C, Tufvesson E, Anderson SD, et al. Effect of inspired air conditions on exercise-induced urinary CC16 levels in athletes. J Appl Physiol 2011; 111: 1059-1065.

8 Beuther DA, Martin RJ. Efficacy of a heat exchanger mask in cold exercise-induced asthma. Chest 2006; 129: $1188-1193$.

9 Brenner AM, Weiser PC, Krogh LA, et al. Effectiveness of a portable face mask in attenuating exercise-induced asthma. JAMA 1980; 244: 2196-2198.

10 Millqvist E, Bengtsson U, Löwhagen O. Combining a beta2-agonist with a face mask to prevent exercise-induced bronchoconstriction. Allergy 2000; 55: 672-675.

11 Nisar M, Spence DP, West D, et al. A mask to modify inspired air temperature and humidity and its effect on exercise induced asthma. Thorax 1992; 47: 446-450.

12 Stewart EJ, Cinnamond MJ, Siddiqui R, et al. Effect of a heat and moisture retaining mask on exercise-induced asthma. BMJ 1992; 304: 479-480.

13 Kennedy MD, Davidson WJ, Wong LE, et al. Airway inflammation, symptoms, and skiing. Scand J Med Sci Sports 2016; 26: 835-842.

14 Frischhut C, Kennedy MD, Niedermeier M, et al. Effects of a heat and moisture exchanger on respiratory function and symptoms post-cold air exercise. Scand J Med Sci Sports 2019; 30: 591-601.

15 Parsons JP, Hallstrand TS, Mastronarde JG, et al. An official American Thoracic Society clinical practice guideline: exercise-induced bronchoconstriction. Am J Respir Crit Care Med 2013; 187: 1016-1027.

16 Quanjer PH, Tammeling GJ, Cotes JE, et al. Lung volumes and forced ventilatory flows. work group on standardization of respiratory function tests. European Community for Coal and Steel. Official position of the European Respiratory Society. Rev Mal Respir 1994; 11: Suppl. 3, 5-40.

17 Anderson SD, Kippelen P. Assessment of EIB: what you need to know to optimize test results. Immunol Allergy Clin North Am 2013; 33: 363-380.

18 Birring SS, Prudon B, Carr AJ, et al. Development of a symptom specific health status measure for patients with chronic cough: Leicester Cough Questionnaire (LCQ). Thorax 2003; 58: 339-343.

19 Spinou A, Birring SS. An update on measurement and monitoring of cough: what are the important study endpoints? J Thorac Dis 2014; 6: S728-S734. 
Dweik RA, Boggs PB, Erzurum SC, et al. An official ATS clinical practice guideline: interpretation of exhaled nitric oxide levels (FeNO) for clinical applications. Am J Respir Crit Care Med 2011; 184: 602-615.

21 Miller MR, Hankinson J, Brusasco V, et al. Standardisation of spirometry. Eur Respir J 2005; 26: 319-338.

22 Crapo RO, Casaburi R, Coates AL, et al. Guidelines for Methacholine and Exercise Challenge Testing-1999. Am J Respir Crit Care Med 2000; 161: 309-329.

23 Ansley L, Stone M, Hull JH. Predicting work rate for exercise-induced asthma exercise challenge. In: XXIX EAACI Congress of the European Academy of Allergy and Clinical Immunology. Allergy 2010; 65: Suppl. s92, 1-790.

24 Birring SS, Matos S, Patel RB, et al. Cough frequency, cough sensitivity and health status in patients with chronic cough. Respir Med 2006; 100: 1105-1109.

25 Birring SS, Fleming T, Matos S, et al. The Leicester Cough Monitor: preliminary validation of an automated cough detection system in chronic cough. Eur Respir J 2008; 31: 1013-1018.

26 Anderson SD, Caillaud C, Brannan JD. Beta2-agonists and exercise-induced asthma. Clin Rev Allergy Immunol 2006; 31: 163-180.

27 Fukuhara A, Saito J, Birring SS, et al. The assessment of asthma with a novel 24-hour cough frequency monitor. Eur Respir J 2016; 48: Suppl. 60, PA1091.

28 Rhee H, Belyea MJ, Sterling M, et al. Evaluating the validity of an automated device for asthma monitoring for adolescents: correlational design. J Med Internet Res 2015; 17: e234.

29 Hull JH, Dickinson JW, Jackson AR. Cough in exercise and athletes. PulmPharmacol Ther 2017: 47: 49-55.

30 Aaron SD, Vandemheen KL, FitzGerald JM, et al. Reevaluation of diagnosis in adults with physician-diagnosed asthma. JAMA 2017; 317: 269-279.

31 Millqvist E, Bake B, Bengtsson U, et al. Prevention of asthma induced by cold air by cellulose-fabric face mask. Allergy 1995; 50: 221-224. 